\title{
Introduction
}

\section{Une question vitale. Connaître, protéger, exposer la vie}

\author{
Joëlle Vailly, Jörg Niewöhner et Janina Kehr
}

«Un enfant naît suite à une procédure de fertilisation in vitro pour un coût de 50000 dollars. A l'autre bout du monde, une jeune mère, très pauvre, meurt du sida ». Ainsi débute, à peu de choses près, une revue de la littérature anthropologique récente traitant de la vie à ses marges, c'est-à-dire lorsque celle-ci commence ou prend fin [Kaufman et Morgan, 2005]. Si elle peut paraître simplificatrice, la juxtaposition de ces deux événements discordants - la naissance en conditions de haute technicité d'un côté, la mort dans le dénuement de l'autre, - présente l'avantage de faire entrer de plain-pied dans la question de la vie en sociétés, autrement dit de la vie des êtres humains au sein de configurations sociales et politiques données. Ils suggèrent en outre des distinctions sémantiques car, à y regarder de plus près, ces deux scènes illustrent des sens différents du terme de vie.

Celui-ci peut d'abord être défini par ce qu'il ne désigne pas. D'une part la vie s'oppose à tout ce qui en est dépourvu - la matière, l'inerte - et distingue le vivant du non-vivant; d'autre part la vie s'oppose à la mort, ce qui, dans le cas des êtres humains soulève des problèmes d'autres natures, avec des implications à la fois sociales, philosophiques, psychologiques, etc. [Ardoino, 2001]. Dans la même optique, l'historien et philosophe des sciences Georges Canguilhem distingue deux sens, le vivant (au sens d'organisation de la matière) et le vécu (au sens d'expérience des êtres humains). Même si Canguilhem consacre surtout ses travaux au vivant, il s'attache à relier en partie vivant et vécu. En témoigne sa proposition de notion «d'allures de vie », qui semble déborder du cadre étroit des molécules et des cellules pour entrer dans celui de la douleur et de la vie quotidienne. Selon cette notion, la maladie serait une allure de vie réglée par des normes vitalement inférieures ou dépréciées [Canguilhem, 1966, p. 137]. Plus généralement, le vivant et le vécu dessinent des espaces vitaux différents mais chevauchants. Ces chevauchements restituent son épaisseur à la notion de vie, car ils permettent de mettre d'autant mieux en relief des enjeux à la fois scientifiques, politiques et moraux. A l'appui de cette idée, João Biehl [2005] explore l'exemple de Catarina, une femme qui incarne les effets réciproques des sciences biomédicales, du gouvernement des vivants, de l'expérience du sida et de la misère dans des zones d'abandon et de mort au Brésil. La vie dont nous l'entendons n'est d'ailleurs pas seulement celle de la vie des malades, elle peut être aussi celle de la vie sociale et politique dans son articulation et sa coproduction avec la vie biologique. La présentation un peu plus loin de différents concepts autour de la vie permettra d'aller plus avant dans cette explicitation sémantique. Voyons d'ores et déjà comment les deux scènes citées en exergue sont partie prenantes de dynamiques sociales marquantes de la fin du $\mathrm{XX}^{\mathrm{e}}$ siècle qui participent activement des évolutions des enjeux autour de la 
vie.

En premier lieu, il convient de souligner le développement galopant des sciences de la vie et de la biomédecine. Après la deuxième guerre mondiale en effet, s'est développé, plus particulièrement en Europe et aux Etats-Unis, un processus de biomédicalisation, c'est-à-dire de coproduction des approches technoscientifiques liées aux sciences de la vie d'une part et des pratiques cliniques d'autre part, qui a connu une grande accélération à partir des années 1980 [Clarke, Mamo, Fisman, Foskett et coll., 2003]. Lors d'un éloge à Canguilhem, Michel Foucault explique d'ailleurs qu' «il a été impossible de constituer une science du vivant sans que soit prise en compte, comme essentielle à son objet, la possibilité de la maladie, de la mort, de la monstruosité, de l'anomalie et de l'erreur » [Foucault, 2001, p. 1591]. Formulé autrement, et ceci reste assurément toujours valable, la compréhension des phénomènes biologiques liés au vivant a à la fois alimenté et été alimentée par l'étude des maladies, notamment génétiques. En lien avec ceci, des changements importants dans le savoir génétique, depuis l'élucidation de la structure de l'ADN au début des années 1950, ont contribué à une molécularisation croissante de la compréhension du biologique et ont consolidé la position clé de la technologie dans la production du savoir scientifique. Celle-ci s'est illustrée par le séquençage du génome humain au tournant du millénaire, qui avait l'objectif, selon la formule emphatique employée par des biologistes à l'époque, de déchiffrer «le livre de la vie ».

Aujourd'hui, avec l'ère de la post-génomique, tout en intégrant des degrés supérieurs de complexité, la molécularisation du vivant se poursuit. De vastes projets de recherche sur les interactions entre gènes et environnement pour comprendre l'étiologie de maladies complexes comme les maladies cardiovasculaires ou le diabète sont développés. Une industrialisation des plateformes de recherche biologique voit le jour, avec la mise sur pied de complexes technoscientifiques imbriqués dans de vastes enjeux financiers. Ceux-ci conduisent des anthropologues à évoquer la constitution d'un «biocapital » [Rajan, 2006], y compris dans les pays dits émergents qui misent une part de leur développement sur les biotechnologies. En outre, le savoir issu des sciences de la vie modifie l'objet de ce savoir (le vivant), comme l'atteste l'annonce de la première reconstitution d'un organisme vivant à partir d'un génome entièrement artificiel aux Etats-Unis ${ }^{1}$. Il ne s'agit ainsi plus seulement de décrire, de subir et d'élucider les mécanismes du vivant, mais de le transformer par des interventions au niveau moléculaire.

Si le développement de la biologie moléculaire joue un rôle crucial dans l'attention portée au vivant, il est important de ne pas ignorer les autres facettes des savoirs sur la vie. La santé publique et l'épidémiologie notamment jouent un rôle aussi crucial, dans bien des secteurs, sur la manière d'influer sur la vie quotidienne, par l'attention aux facteurs de risque tels que les modes de vie ou le contrôle de la dissémination des maladies infectieuses par les vaccinations et l'attention portée à la vie sociale. Des approches liées à la prévention et au risque sur des populations entières sont développées, produisant des inversions temporelles entre les symptômes et le fait de prendre garde à sa santé (veiller à son régime alimentaire avant de connaître des problèmes sérieux, etc.). Plus précisément, les pratiques de dépistage, d'anticipation et de préven-

\footnotetext{
${ }^{1}$ Cf. l'article intitulé « Création d'une cellule « synthétique » » (Le Monde du 22.5.10).
} 
tion de la maladie s'adressent aux populations et à chacun simultanément. Chacun à l'intérieur d'une population donnée est conduit à se faire dépister quand cela est possible et à adopter un comportement qui préserve des risques de maladie. La psychiatrie montre également une tendance à étendre ses catégories d'analyse, et de nombreuses formes de vie sont aujourd'hui comprises dans un répertoire psychologique et psychiatrique, comme dans les cas de formes d'anxiété ou d'hyperactivité. C'est dire que la biomédicalisation, au sens large, se manifeste également de manière moins sensationnelle qu'avec la transformation du vivant, mais tout aussi prégnante, en étendant les domaines de la vie sous sa juridiction.

En second lieu, la vie des personnes et leur état de santé peuvent être considérés à l'aulne des effets des échanges transnationaux croissants et de la mondialisation, dans un contexte où, depuis les années 1980-1990, les échanges globaux de savoirs, de marchandises et d'individus connaissent une explosion. Ces assemblages globalisés créent aujourd'hui des relations puissantes, des dépendances et des opportunités entre les Etats-nations et les continents [Ong et Collier, 2005], qui influent non seulement sur les modes de gouvernement et de régulation, mais aussi sur la vie des personnes et les questions d'appartenance et d'identités. Nous n'en mentionnerons ici que quatre aspects. D'abord, cette circulation internationale se manifeste dans le domaine de la biomédecine par le brassage des idées, des connaissances, des méthodes et des matériaux biologiques. L'expansion du rôle d'Internet et les possibilités croissantes de mobilité pour les patients participent de ce processus. La fragmentation du vivant, qui est décomposé en objets stabilisés, congelés, vendus et échangés, combinée à la mondialisation confère à ces éléments un caractère de plus en plus transnational [Rose, 2008]. En outre, en lien également avec la mondialisation, des maladies infectieuses anciennes ou nouvelles - dont beaucoup, comme la tuberculose, la poliomyélite ou différents types de grippes, paraissaient éradiquées dans les pays dits du Nord depuis la transition épidémiologique du premier tiers du $\mathrm{XX}^{\mathrm{e}}$ siècle - semblent apporter, de nouveau, des menaces sur la santé des populations de ces pays. Soulignons toutefois que bien souvent, il y a lieu de distinguer la réalité des problèmes sanitaires de leur perception par les populations et les gouvernants, car, comme le soulignent Zoé Vaillant et Gérard Salem [2008, p. 5], « même si, à l'échelle mondiale, les espaces peuvent sembler facilement interdépendants et faciliter en cela la diffusion des maladies, l'inscription territoriale des faits de santé demeure déterminante dans la diffusion ou non des maladies, des comportements à risque, etc. ». Ensuite, la mondialisation économique, les flux de personnes, de capitaux et d'information, les inégalités entre pays riches et pays pauvres ou entre mondes sociaux, et l'urbanisation croissante ont un impact sur la santé des populations [Vaillant et Salem, 2008]. L'accès aux médicaments antipaludiques ou les traitements les plus efficaces contre le sida constituent deux exemples de ce réseau d'échange certes serré, mais qui met ceux et celles qui ne peuvent prendre part à sa coproduction et qui ne peuvent bénéficier de ses avantages dans des positions menaçantes pour leur vie, alors exposée aux dangers. Enfin, les inégalités associées à cette mondialisation contribuent de manière significative à une autre mobilité internationale, celle de la migration sous contrainte économique ou politique. Les mouvements de personnes à travers les frontières conduisent à une pléthore de nouvelles institutions visant à les contenir et à des formes de régulation qui se traduisent par de nouvelles normes légales en matière de migration. Ces dispositifs atti- 
rent l'attention des chercheurs en sciences sociales, non seulement sous l'angle symbolique et politique, mais aussi dans la dimension de la lutte pour la vie qu'elles constituent.

Tout en connaissant ainsi aujourd'hui des transformations marquantes, ces dynamiques sociales autour du vivant et de la vie s'ancrent dans des processus historiques. L'idée que la vie puisse être étudiée, et peut-être comprise, tient d'abord évidemment à sa constitution en tant que concept. Ce n'est pas avant la fin du XVIII ${ }^{\mathrm{e}}$ siècle qu'une division fondamentale s'est formée entre vivant et non-vivant, et que les êtres ont été séparés des choses. Comme l'explique François Jacob dans son histoire de la biologie, ce qui a donné leurs propriétés aux êtres, c'est l'organisation cachée derrière la structure visible. A pu alors apparaitre l'idée d'un ensemble de qualités qui leur est particulières, qu'appellera vie le XIX ${ }^{\mathrm{e}}$ siècle [Jacob, 1970, p. 53]. Historiquement, le regard scientifique et médical a créé les conditions par lesquelles la vie et la mort ont pu être ainsi appréhendées et constitués comme des processus fondamentalement biologiques [Kaufman et Morgan, 2005]. Les théories darwiniennes de l'évolution et les concepts formés par la physiologie au XIX ${ }^{\mathrm{e}}$ siècle ont permis l'expression des sciences de la vie qui ont connu l'explosion et les transformations radicales dont il a été question plus haut avec la biologie moléculaire. Mais il serait évidemment erroné de penser uniquement l'enjeu de la vie à la lumière des développements de la biologie, car son émergence est liée également, et de façon fondamentale, au politique. Parce qu'elles pointent l'entrée de la vie et de la santé dans les stratégies politiques, les théories proposées par Michel Foucault, aujourd'hui relativement connues, livrent des clés d'analyse fécondes.

D'abord, la notion de biopouvoir renvoie à l'émergence d'un pouvoir sur la vie : « Ce qui s'est passé au XVIII ${ }^{\mathrm{e}}$ siècle dans certains pays d'Occident, et qui a été lié par le développement du capitalisme [...], ce fut rien moins que l'entrée de la vie dans l'histoire - je veux dire l'entrée des phénomènes propres à la vie de l'espère humaine dans l'ordre d'un savoir et d'un pouvoir -, dans le champ des techniques politiques » [Foucault, 2000, p. 186]. Ce pouvoir s'est déployé au plan individuel et, un peu plus tard, collectif. Individuel, parce qu'il était centré sur le corps, pour le dresser et majorer ses forces, en le débarrassant d'habitudes corporelles, sexuelles, sociales qui auraient pu porter préjudice à la santé ; collectif, parce qu'il s'attachait à contrôler l'espèce, qui est entrée comme enjeu dans les stratégies politiques pour assurer prolifération et longévité, en s'appuyant sur la démographie et l'épidémiologie. Ces deux volets, appelés « anatomo-politique du corps humain » et «bio-politique de la population » constituaient deux pôles, qui pouvaient d'ailleurs se croiser. En somme, les politiques de santé se sont orientées autour d'un appareil prenant en charge les malades et d'un dispositif permettant d'observer, de mesurer et d'améliorer l'état de santé de la population [Foucault, 2001, p. 731]. Les rapports entre la vie et le politique sont liés non seulement à l'émergence de rationalités scientifiques et médicales telles qu'elles ont été décrites plus haut (sans parler de projets pseudo-scientifiques tels que l'eugénisme de Galton), mais aussi à l'utilisation de statistiques, la mise en place d'une l'hygiène urbaine, le déplacement des cimetières aux périphéries des villes, la surveillance des corps dans les écoles et les prisons, etc. Dès lors, les stratégies politiques se sont attachées à gérer la vie biologique, non seulement en tant que force de travail indispensable au capitalisme, mais comme élément d'une bio-histoire au cours 
de laquelle la possibilité scientifique de transformer la vie, de l'évaluer et de l'améliorer, était recherchée. Comme en miroir, elles ont semblé laisser de côté la question de la mort et des mourants, sauf, exception notable, lorsqu'elles se retournent sous forme de thanatopolitiques (politiques de mort) sur des bases génocidaires et/ou racistes. Il convient de noter au demeurant que certains auteurs considèrent que l'entrée de la vie dans les stratégies politiques est bien plus ancienne [Agamben, 1997] ou dépend de mécanismes généraux que l'on retrouve à d'autres périodes de l'histoire et en d'autres lieux, comme dans la Rome antique [Fassin, 1996, p. 227].

Ce bref détour historique permet de mieux saisir l'enjeu que recèle la vie dans les sociétés actuelles. Dans cet ordre d'idée, le sociologue Nikolas Rose [2008] rappelle que pour Max Weber, les êtres humains conduisent leur vie par une sotériologie, autrement dit une doctrine du salut qui donne un sens à la souffrance, lui trouve des raisons et des moyens de s'en délivrer. Selon Rose, notre sotériologie prend de plus en plus une forme somatique, car nous identifions et interprétons beaucoup de nos ennuis en termes de santé et de vitalité 2 . Ajoutons que tout se passe comme si la préservation d'une vie en bonne santé était devenue une sorte de vertu, avec toute la dimension morale que celle-ci peut drainer: il deviendrait moralement répréhensible de ne pas prendre soin de sa santé et de ne pas prévenir la souffrance de ses proches. Toutefois, on aura compris à travers les éléments évoqués plus haut qu'il faut restreindre cette constatation aux catégories de populations qui ont les ressources et les capacités requises pour préserver cette vie en bonne santé : la technicisation de la médecine dans les pays riches n'obère évidemment pas la stratification liée aux inégalités socioéconomiques, aussi bien au sein de ces pays que par comparaison aux pays pauvres. La notion de «bio-légitimité » proposée par Didier Fassin [2009] permet de rendre compte de cette restriction. En effet, elle met en évidence la place centrale qu'occupe la santé au sein de nos sociétés tout en prenant en compte la question des inégalités dans la valeur accordée aux vies. Elle permet ainsi de moduler l'idée d'un pouvoir globalisant qui s'exercerait de façon indiscriminée en faveur de toutes les vies quelles qu'elles soient. En soulignant le sens et la valeur de la vie plutôt que les stratégies de contrôle, la bio-légitimité définit un pouvoir de la vie plutôt qu'un pouvoir sur la vie [Fassin, 2009].

Ces différents points - biomédicalisation, mondialisation, histoire du concept de vie, entrée dans le politique et valeur attachée à la santé - dessinent le contexte dans lequel nous inscrivons nos recherches. Avant de les présenter plus précisément, nous souhaitons opérer un détour par les travaux de chercheurs en sciences sociales et humaines s'intéressant à la vie.

Au cours des dernières années, quatre lectures de la notion de vie sur lesquelles nous souhaitons ici nous attarder - sans viser à l'exhaustivité - ont été développées. Au-delà de leur commune référence à Foucault, elles touchent à des thèmes de recherche différents et elles ont implications diverses. La première émane d'écrits politiques et philosophiques, notamment italiens, alors que les trois autres ont été proposées aux intersections de la sociologie et de l'anthropologie, particulièrement en Grande-Bretagne, aux Etats-Unis et en France.

Avec une approche philosophique, Giorgio Agamben [1997] s'appuie sur le phé-

2 Un exemple parmi d'autres repose sur l'extension de catégories de la santé mentale dont il a été question précédemment. 
nomène du camp de la mort pour développer l'idée que les biopolitiques contemporaines visent à produire une "vie nue » réduite à son essence biologique et des citoyens dans le corps naturel desquels est en jeu leur être politique même : «La politique ne connaît aujourd'hui aucune autre valeur [...] que la vie [...]. La possibilité de faire le partage entre notre corps biologique et notre corps politique, entre ce qui est incommunicable et muet et ce qui est communicable et exprimable, nous a été enlevée une fois pour toutes », dit-il [Agamben, 1997, p. 18 et 202]. Ces travaux peuvent être rapprochés à certains égards de ceux de Michael Hardt et Antonio Negri [2000] du fait de leur approche philosophique commune et de leur caractère généralisateur. Selon Hardt et Negri, le passage au biopouvoir a eu lieu non au $\mathrm{XVII}^{\mathrm{e}}$ et $\mathrm{XVIII}^{\mathrm{e}}$ siècles comme l'avance Foucault, mais dans les années 1970. Il aurait été promu au nom des multinationales et des organisations transnationales en produisant une organisation en réseau qui dépasse les Etats-nations et qu'ils nomment «empire ». Malgré leur apport théorique, les travaux d'Agamben, Hardt et Negri ont attiré des critiques, particulièrement de la part d'anthropologues et d'adeptes des science and technology studies (STS) en raison de leur occultation des différenciations importantes dans les moyens et les fins entre les biopolitiques, et en particulier entre les atrocités nationales-socialistes en tant que paradigme biopolitique et les politiques contemporaines. Selon ces critiques, au lieu de subsumer dans une macro théorie des catégories et des processus divers, il serait plus pertinent de les analyser de façon différenciée et nuancée [Rabinow et Rose, 2006 ; Agier, 2002].

Une deuxième approche met au centre de l'analyse la notion de "vie elle-même » (life itself). Cette grille d'analyse a été développée à partir du constat que les modes actuels de gouvernement n'ont plus seulement pour objectif de développer la santé et de contenir la maladie, mais de cibler directement les capacités vitales et d'en améliorer les qualités [Franklin, 2000 ; Rose, 2001 ; Rose, 2007]. Nikolas Rose soutient l'idée que l'émergence des sciences de la vie rend possible un nouvel accès moléculaire à la vie elle-même : "Les êtres humains en viennent à faire une expérience d'eux-mêmes inédite en tant que créatures biologiques; et en tant qu'êtres biologiques, leur existence vitale devient un enjeu de gouvernement, la cible de nouvelles formes d'autorité et d'expertise, un domaine de connaissance hautement chargé, un territoire d'exploitation bioéconomique en expansion, un principe éthique organisateur et l'objet même de la politique moléculaire de la vie » [Rose, 2007, p. 4] $]^{3}$. Il s'appuie prioritairement sur l'analyse de la génétique, ou plus précisément sur les technologies de recherche génétique, les diagnostics et les thérapies développées - ou au moins promises - en lien avec le séquençage du génome humain. Empruntant le terme à Adriana Petryna [2002] à propos des personnes exposées aux radiations de Tchernobyl, il évoque une «citoyenneté biologique », qui rend compte des projets menés par les citoyens et liés directement à leur existence biologique d'êtres humains, déclinée en individus, hommes et femmes, membres de familles et de lignées, de communautés, de populations et d'espèce.

Alors que l'analyse consistant à dire que la vie elle-même émerge au sein des biopolitiques est partagée par beaucoup de commentateurs, une critique - ou au moins un élargissement critique - digne d'attention contre la nature monolithique des politiques

\footnotetext{
${ }^{3}$ C'est nous qui traduisons.
} 
de la vie-même a été formulée. En particulier, des chercheurs issus des STS ont développé l'idée que la notion de molécularisation, tout en étant très pertinente sur les bases empiriques que Rose et ses collègues dessinent, ne couvre pas la diversité de la production du savoir scientifique et les formes prises par le biopouvoir [Raman et Tutton, 2010]. Ils suggèrent en la matière de prendre en compte non seulement les individus mais également les populations, non seulement la responsabilité des personnes mais aussi les mécanismes de contrôle et de surveillance plus anciens. Ils soulignent que «l'existence biologique des êtres humains est politisée par le biais de plusieurs discours complémentaires et rivaux portant sur les thérapies médicales, par des choix opérés en début et fin de vie, la santé publique, l'environnement, la migration et le contrôle des frontières, impliquant de multiples politiques de la vie, plutôt qu'une seule » [Raman et Tutton, 2010, p. 1] $]^{4}$. C'est pourquoi ces auteurs soutiennent l'idée d'une « multiplicité des politiques de la vie» (multiple politics of life).

Au-delà de cette multiplicité, des déplacements substantiels autour du concept foucaldien de biopouvoir et de biopolitiques ont été proposés par Didier Fassin [2009]. Tout d'abord, est mis en lumière le fait que les biopolitiques telles qu'analysées par Michel Foucault sont des politiques axées davantage sur les populations que sur la vie. Plus généralement, ce dernier ne consacre pas réellement ses travaux à la vie, mais plutôt à la façon dont les êtres vivants ont été considérés en tant que populations et individus, et dont la gouvernementalité et la subjectivation (i.e. la constitution de sujets, avec leurs aspirations, leurs besoins, leurs choix, etc.) ont façonné notre vision du monde et de l'humanité [Fassin, 2009]. Or, la focalisation sur l'art de gouverner et sur les processus de subjectivation tend à marginaliser le sens et les conséquences des pratiques de gouvernement pour la vie des personnes. En parallèle à l'étude des politiques de la vie-même telle qu'elles ont été présentées plus haut, Didier Fassin propose de développer une autre anthropologie de la vie, en s'attachant à étudier la vie vécue comme corps (pas seulement comme cellules) et comme société (pas seulement comme espèce). Cette vie, que l'auteur propose d'appeler la vie en tant que telle (life as such), est conçue comme l'ensemble des événements allant de la naissance à la mort, qui peut être prolongée par des politiques sanitaires et sociales, mais aussi écourtée par la violence, etc. Sur la base de différentes données sur les régimes de vie [Collier et Lakoff, 2005] rassemblées dans des recherches soucieuses des implications des politiques dans les pays du Sud ou chez des populations marginalisées, la vie en tant que telle focalise ainsi notre attention sur les conséquences sociales, matérielles et morales des politiques de la vie et des pratiques associées - une perspective que Didier Fassin puise davantage chez Hannah Arendt et Walter Benjamin que chez Michel Foucault.

La diversité des objets traités au sein des sciences sociales et humaines analysant la vie reflète ainsi une grande variété des thématiques, la polysémie du terme de vie pouvant renvoyer aussi bien aux techniques de procréation assistée et aux politiques de santé publique qu'à la vie dans les camps, au génocide, à la violence physique, etc. Notre contribution à ce champ d'étude, quant à elle, restreint ses analyses à la santé, à la biomédecine et aux technologies associées. Toutefois, il convient de souligner que l'ouvrage que nous coordonnons confère une place aussi cruciale au social et au poli-

\footnotetext{
${ }^{4}$ C'est nous qui traduisons.
} 
tique qu'au médical ou au biologique, en tentant d'en analyser les articulations et les coproductions. Il traite ainsi de la coproduction contemporaine des vies sociales et biologiques des êtres humains, aussi bien du point de vue de leurs fondements épistémiques que de leurs effets politiques et des valeurs sur lesquelles elles reposent. Il cherche à montrer comment les savoirs, les techno-sciences, les politiques de santé et les expériences de la maladie façonnent la vie, modifient ses conceptions, gèrent les disparités sociales et produisent (ou non) de la différence. Des tensions entre innovations techniques et persistance de régimes médico-sociaux anciens, entre politiques de santé indiscriminés et inégalités sociales, entre particularités locales et circulation des savoirs pourront se faire jour.

Tout en nous appuyant sur le corpus qui vient d'être brièvement présenté, nous souhaitons procéder à certains déplacements de perspective pour ce qui concerne le champ de la santé, de la biomédecine et de la vie selon trois directions :

1. en intégrant davantage les enjeux politiques à l'analyse que cela n'a été fait dans certaines études s'intéressant surtout aux sciences et aux techniques. Ces enjeux incluent non seulement la constitution des sujets, mais aussi la place des populations marginalisées ou défavorisées dans ces dispositifs vitaux, la construction ou la transformation des identités nationales ou régionales, ainsi que la question des migrations et des contrôles de frontières. Il s'agit de fait de prendre en compte aussi bien la question de la responsabilité des citoyens envers leur propre santé que les rapports de pouvoir et leurs effets en fonction des différentes positions sociales, dans les discours et au plus près des pratiques.

2. en analysant les vies quotidiennes et les expériences des malades (ou des personnes) et de leurs proches, ainsi que les pratiques quotidiennes des soignants, avec l'objectif de conférer une place centrale au vécu tout en étudiant les liens entre vivant et vécu. D'un point de vue disciplinaire, il s'agirait en somme de dépasser la césure entre les approches classiques de la sociologie et de l'anthropologie de la santé d'un côté et les science and technology studies de l'autre, en prenant au sérieux la question des nouvelles technologies biomédicales, mais sans montrer trop de fascination à leur égard, afin de restituer, comme cela a été dit en introduction, une forme d'épaisseur à la vie en sociétés.

3. en incluant à l'analyse d'autres formes de savoir que la biologie moléculaire et la génétique, quelle que soit la place importante qu'elles occupent aujourd'hui dans le savoir biomédical et la compréhension de nous-mêmes. La place prépondérante de la biologie a parfois conduit des chercheurs en sciences sociales à occulter d'autres approches plus anciennes, caractéristiques au demeurant du biopouvoir, comme l'épidémiologie, la santé publique et les statistiques, qui participent toujours, elles aussi, de la façon dont la vie est conceptualisée, transformée, objet de valeurs et de politiques. Autrement dit, tout en explorant de nouvelles étapes de la recherche biomédicale et des sciences de la vie, il s'agit de contribuer à rendre compte de la diversité des situations, dès lors qu'elles ne relèvent pas des nouvelles techniques du vivant.

Mobilisant des approches anthropologiques et sociologiques, cet ouvrage est pleinement ancré dans les pratiques. Les recherches sur lesquelles il s'appuie reposent sur des entretiens et/ou des observations ethnographiques, aussi bien auprès de professionnels de santé, de responsables d'administration de santé ou d'associations, que de malades et leur famille. Elles reposent également sur l'analyse de discours de la littéra- 
ture scientifique et des médias, ainsi que sur la littérature grise. Ces diverses sources constituent, au sens propre, des points de vue différents adaptés aux questions posées dans chaque chapitre. Elles donnent à voir aussi bien l'expérience des malades que les savoirs autour de la maladie, les discussions politiques ou les interrogations éthiques. Elles cherchent à lier les processus microsociaux aux contraintes structurelles plus globales, d'ordre économique, politique ou social. Si les enquêtes ont été conduites principalement en Europe, le choix a été de débuter l'ouvrage par une enquête menée en Afrique du Sud, qui permet d'ouvrir une perspective élargie sur la vie dans le monde contemporain et prémunit d'emblée contre une vision centrée sur les contextes européens ou nord-américains. Toutes les autres enquêtes ont été menées surtout en France et en Allemagne, mais aussi à Chypre et en Angleterre, sans avoir, dans la plupart des cas, une dimension comparatiste explicite.

L'ouvrage s'organise en onze chapitres distribués en deux parties. La première partie est plus particulièrement orientée vers les enjeux politiques, en lien d'une part avec la vie vécue de malades, d'autre part avec les nouvelles technologies du vivant. Plus précisément, les deux premiers chapitres traitent de l'articulation entre existence, place du politique et éthique. A partir du dernier entretien de Jacques Derrida sur le thème de la survie et en se basant sur l'expérience de malades atteints du sida en Afrique du Sud, Didier Fassin cherche à dépasser la dualité vie biologique/vie politique que beaucoup de chercheurs et d'étudiants considèrent comme heuristique. En défendant une position non seulement théorique, mais également éthique, il réhabilite en somme le «simple fait de vivre », au sens de Walter Benjamin, en tant que vie bonne. Avec une approche ethnographique également, Stefan Beck, qui a conduit une recherche sur les dons de moelle osseuse à Chypre, explique comment la vie des malades passe audessus des réticences politiques, biologiques, culturelles et sociales entre les communautés turques et grecques de l'île. Son enquête est l'occasion de mener une réflexion sur les agencements sociaux, les identités et les liens générés par la biomédecine dans et au-delà des Etats-nations, qui combinent sciences, politique et éthique. Dans les trois chapitres suivants, en mobilisant l'étude de documents, nous tentons de rendre compte d'enjeux également politiques et éthiques, mais cette fois autour des nouvelles technologies du vivant. Boris Hauray analyse la manière dont le problème des recherches sur l'embryon est construit, thématisé et interprété en France, en Allemagne et au Royaume-Uni, en s'appuyant sur une analyse des discours officiels. Il montre qu'en même temps que ce problème est saisi par les pouvoirs publics et donne lieu à des discussions publiques, il semble faire l'objet d'une dépolitisation du fait de sa mise à l'écart d'un débat qui serait proprement politique. Bertrand Pulman s'intéresse aux nouvelles techniques en matière de procréation et aux pratiques associées, afin d'analyser leur impact grandissant sur les relations sociales et sur nos représentations de nous-mêmes. Tout en informant largement sur l'état de ces techniques et en faisant le point des expériences déjà réalisées, il présente certains des débats et des enjeux à ce sujet, en particulier autour de la notion d'eugénisme libéral. Thomas Lemke et Malaika Rödel s'intéressent quant à eux au recours au test $\mathrm{ADN}$ dans les procédures d'immigration à des fins de regroupement familial en Allemagne, en présentant tout 
d'abord son contexte historique et son importance actuelle. Ils montrent ensuite certaines de ses conséquences et les problèmes juridiques, sociaux et techniques qu'il pose. Enfin, ils discutent ces pratiques à l'aulne des thèmes de la « génétisation » de la société et de la « citoyenneté biologique ».

Dans la deuxième partie, nous nous attachons à étudier la vie du point de vue des pratiques dans les domaines de la clinique et de la santé publique. Les trois premiers chapitres s'appuient sur des enquêtes ethnographiques dans des contextes hospitaliers, afin d'analyser des pratiques cliniques, des expériences de malades ou le cas des patients en fin de vie. S'appuyant sur une enquête menée dans un hôpital de la région parisienne, Joëlle Vailly étudie la façon dont est poursuivi l'objectif de garder non seulement une «qualité de vie», mais aussi ce qu'elle propose d'appeler une «vie de qualité », à partir des pratiques de dépistage pour une maladie génétique (la mucoviscidose). Elle montre la façon dont les manières de repérer la maladie avant et après la naissance interfèrent et créent une tension autour du seuil anthropologique de la naissance. Katrin Amelang interroge les modes de définition de la normalité dans la vie des malades après transplantation, grâce à une étude menée dans un service de transplantation du foie. Elle en présente quatre définitions, allant des analyses chimiques au quotidien perçu comme «normal » par le malade, en passant par les usages cliniques des analyses et l'expérience des patients. L'étude permet de mesurer la variabilité de cette définition, entre molécules et vie quotidienne. Enfin, en s'appuyant sur une enquête ethnographique dans un service des Urgences de la région parisienne, Carine Vassy et Marie-France Couilliot étudient l'impact de la politique hospitalière et des contraintes organisationnelles et gestionnaires sur la prise en charge des patients en fin de vie, et sur la définition de ce qu'est un patient mourant. Elles montrent que la prise en charge des personnes en fin de vie, si elles ne décèdent pas très vite, ne semble $\mathrm{y}$ être du ressort de personne. Enfin, les trois derniers chapitres s'attachent à mettre en évidence le rôle de la vie sociale dans l'élaboration des savoirs et des pratiques dans le domaine de la santé publique. Sur la base d'une enquête ethnographique sur les recherches en laboratoire et sur les programmes de prévention dans les jardins d'enfants, Jörg Niewöhner et Michalis Kontopodis analysent la pertinence des concepts de molécularisation, optimisation et subjectivation, proposés par Nikolas Rose, dans le cas des maladies cardiovasculaires. Tout en confirmant celle-ci, ils l'infléchissent pour montrer l'émergence d'une nouvelle ouverture du corps et le retour de conceptions plus paternalistes du soin. Janina Kehr analyse la manière dont la vie sociale des malades, rarement au centre de l'intérêt en biomédecine, est appréhendée par les professionnels de santé dans un centre de lutte anti tuberculose. Elle montre que lors des «enquêtes autour d'un cas », cette vie sociale n'est pas seulement façonnée comme somme de relations infectieuses, mais également comme quotidien «épais », faisant de ce dépistage une pratique rejoignant les aspirations de la médecine sociale du XIX ${ }^{\mathrm{e}}$ siècle. Susanne Bauer, quant à elle, s'appuie sur des observations et sur des documents afin d'analyser la manière dont «le social» est pris en compte dans les recherches épidémiologiques. Elle décrit les opérationnalisations statistiques et conceptuelles variées du social dans ce domaine de recherche, puis la façon dont les chercheurs procèdent à des translations sur des concepts tels que la prédisposition et la vulnérabilité.

Du point de vue du champ des sciences sociales, il convient de souligner que la littérature existante sur le thème de la vie dans le cadre de la biomédecine est souvent 
anglophone et issue des perspectives de recherche anglaises et nord-américaines. Dès lors, ce livre propose un dialogue avec la recherche anglophone sur le thème de la vie et sur les termes - théoriques et pratiques - dans lesquels il peut être abordé en sciences sociales, tout en cherchant à apporter une perspective nouvelle. En effet, étant issu de la collaboration entre des équipes de recherche françaises et allemandes, le livre véhicule des approches académiques et des contextes aux plans historiques, politiques et éthiques différents des études antérieures 5 . Nous souhaitons de ce point de vue que cet ouvrage puisse inspirer de futures collaborations internationales et comparatives.

D’un point de vue plus général, pour paraphraser Mitchell Dean [1999, p. 99] évoquant les biopolitiques, la question de la vie, telle qu'elle est posée dans cet ouvrage, concerne les conditions sociales, culturelles, environnementales, économiques et géographiques dans lesquelles les êtres humains vivent, procréent, tombent malades, restent ou deviennent en bonne santé, et meurent. Autant de questions qui occupent une place de plus en plus centrale dans les conduites individuelles et collectives [Fassin, 1996]. Dans ce contexte, on l'aura compris, nous avons souhaité que les travaux rassemblés ici touchent aussi bien aux sciences et aux techniques, à la vie dans la cité, à la souffrance et la maladie, qu'à la famille et aux relations sociales quotidiennes. C'est dire qu'en cherchant à dépasser les problèmes circonstanciels ou locaux, l'ouvrage peut apporter des éclairages sur des questions générales telles que les identités individuelles et collectives, la normalité, les valeurs, etc. Nous souhaitons ainsi toucher à des questions anthropologiques et sociologiques qui se posent face aux enjeux biologiques et sociaux majeurs des sociétés actuelles, telle que la gestion de la santé et de la maladie ou les phénomènes de migration, sans pour autant perdre de vue la spécificité de chaque situation et des pratiques analysées ici. C'est ce à quoi cet ouvrage, nous l'espérons, s'emploie.

\section{BIBLIOGRAPHIE}

Agamben G. (1997), Homo sacer : le pouvoir souverain et la vie nue, Seuil, Paris. AGIER M. (2002), «Between war and city: towards an urban anthropology of refugee camps, Ethnography », 3, p. 317-41.

ARdoino J. (2001), «L'humain, le vivant et le vécu », Prétentaine, 14-15, p. 109-116.

BIEHL J. (2005), Vita. Life in a zone of social abandonment, University of California Press, Berkeley, Los Angeles, London.

Clarke A.E., Shim J.K., MAMO L., Fosket J.R., Fishman J.R. (2003). « Biomedicalization: technoscientific transformations of health, illness, and US biomedicine », American Sociological Review, 68, p. 161-94.

\footnotetext{
5 Dans la mesure où les littératures française et allemande sont chacune peu lues dans la langue de l'autre, une version peu différente est publiée en allemand, ce qui donne une visibilité importante de la recherche en cours dans le pays voisin.
} 
CAnguilhem, G. (1966), Le normal et le pathologique, Presses Universitaires de France, Paris.

COLliER S.J. et LAKOFF A. (2005), « On regimes of living », in COLLIER S.J. et A. ONG (dir.) Global assemblages. Technology, politics, and ethics as anthropological problems, Malden, Blackwell Publishing, p. 22-39.

DEAN M. (1999), Governmentality: power and rule in modern society, Sage Publications, London, Thousand Oaks, New Delhi.

FASSIN D. (1996), L'espace politique de la santé. Essai de généalogie, Presses Universitaires de France, Paris.

FASSIN D. (2006), « La biopolitique n'est pas une politique de la vie », Sociologie et Sociétés, 38(2), p. 35-48.

FASSIN D. (2009), «Another politics of life is possible », Theory, Culture \& Society, 26 (5), p. 44-60.

FouCAUlT M. (2000 [1976]), La volonté de savoir. Histoire de la sexualité I, Gallimard, Paris.

FOUCAUlT M. (2001[1994]), Dits et écrits II, 1976-1988, Gallimard, Paris.

FRANKLIN S. (2000), "Life itself: global nature and the genetic imaginary », in FrANKLIN S., LURY C., STACEY J. (dir.), Global Nature, Global Culture, Sage Publications, London.

HARDT M. et NEGRI A. (2000), Empire, Harvard University Press, Cambridge.

JACOB F. (1970), La logique du vivant. Une histoire de l'hérédité, Gallimard, Paris.

KAUFMAN S.R. et MORGAN L.M. (2005), « The anthropology of the beginnings and ends of life », Annual Review of Anthropology, 34, p. 317-341.

ONG A. et COLliER S.J. (2005), Global assemblages: technology, politics, and ethics as anthropological problems, Blackwell Publishing, Malden.

Petryna, A. (2002), Life exposed: biological citizens after Chernobyl, Princeton University Press, Princeton.

RABINOW P. et Rose N. (2006), « Biopower today », Journal of BioSocieties, 1, p. 195-217.

RAJAN S. (2006), Biocapital : the constitution of postgenomic life, Duke University Press, Durham.

RAMAN S. et TUTTON R. (2010), «Life, science, and biopower », Science, Technology \& Human Values, 35(5), p. 711-734.

Rose N. (2001), « The politics of life itself », Theory, Culture \& Society, 18, p. 1-30

ROSE N. (2007), The politics of life itself. Biomedicine, power, and subjectivity in the twenty-first century, Princeton University Press, Princeton.

Rose N. (2008), « The value of life: somatic ethics and the spirit of biocapital », Daedalus, Winter 2008, p. 36-48.

VAillant Z. et SAlem G. (2008), Atlas mondial de la santé. Quelles inégalités? Quelle mondialisation? Editions Autrement, Paris. 